\title{
Propagation of Multipath Components at an Urban Intersection
}

\author{
Kim Mahler*, Wilhelm Keusgen*, Fredrik Tufvesson†, Thomas Zemenћ, Giuseppe Caire' \\ *Wireless Communications and Networks, Fraunhofer Heinrich Hertz Institute, Berlin, Germany \\ $\dagger$ Department of Electrical and Information Technology, Lund University, Lund, Sweden \\ \$ Digital Safety and Security Department, Austrian Institute of Technology, Vienna, Austria \\ 'Communications and Information Theory Group, Technische Universität Berlin, Berlin, Germany
}

\begin{abstract}
Urban intersections constitute an important safetycritical scenario for vehicle-to-vehicle communication. Based on measurements, this paper presents detailed investigations of the radio wave propagation processes in a typical urban intersection. We focus on one time instance of the propagation process and set up hypothesis for locations of the scattering objects. Multipath components (MPCs) are identified and an MPC tracking algorithm is applied. The number of MPCs and the lifetime of MPCs for this communication scenario are analyzed. It is concluded that the lifetime of an MPC is heavily dependent on its relative power, with typical values in the range 0.05-0.3 s corresponding to $0.5-3 \mathrm{~m}$ travel distance of the vehicles.
\end{abstract}

Keywords - Radio propagation, Multipath channels, Intelligent transportation systems, Collision avoidance, Vehicular and wireless technologies

\section{INTRODUCTION}

Inter-vehicular radio communication will play an important role in future collision avoidance systems because of its distinctive capability as a vehicular sensor. Present vehicular sensors, such as radar or video sensors, already enable onboard sensor fusion systems to establish a comprehensive perception of the surrounding. However, radio communication has a unique capability: it enables vehicles to exchange information, even if there is no direct line-of-sight between the vehicles. This means in practice that two vehicles driving towards an intersection on collision course are able to detect each other, even if the line-of-sight is obstructed. Since vehicular communication systems are predominantly envisioned in the $6 \mathrm{GHz}$ band, propagation through massive obstructing objects is not possible and radio communication solely depends on scattering objects at and around intersections. In order to enhance vehicular communication at intersections, researchers focus on a better understanding of the communication performance and its underlying radio propagation process.

Field trials with measurements of packet delivery rates (PDR) and received signal strength were conducted at different types of intersections in [1] and [2]. The measurements were performed with one moving vehicle and one static vehicle at different positions; the results were presented w.r.t. to the position of the moving vehicle. Intervehicular PDR and communication ranges w.r.t. to the positions of both vehicles for different intersection categories are presented in [3]. Channel measurements at different intersection types with typical power delay profiles, pathloss and delay spreads are presented in [4]. It is shown that communication under non-line-of-sight (NLOS) conditions is problematic, but that roadside buildings can create important propagation paths. The scattering objects of the discrete multipath components (MPCs) were determined by drawing a scattering ellipse corresponding to the MPC delay at several time instances. A directional analysis of MPC in different scenarios, including an urban intersection, is introduced in [5]. It is found that in the absence of LOS, first-order reflections from a small number of scattering objects can account for a large part of the received power.

Radio channel measurements are also being used for a validation of propagation models and simulations. Channel sounder data in [6] are aligned with results from a ray tracer by comparing channel gain and power delays profiles. Further, channel measurement results in [7] are compared with a propagation model derived in [8]. For a further improvement of the model, the authors of [7] suggest to include the number of and properties of the available scatterers at the specific intersection into the model.

The contribution of this paper is a comprehensive analysis of scatterers and the temporal behavior of MPCs at an urban intersection. The high time resolution of our delay estimates in combination with an exact positioning system and additional tools allow a reliable identification and localization of scattering objects. In addition, the implemented MPC tracking algorithm enables an analysis of the number of MPCs and the so-called lifetime of MPCs. The results of this paper contribute to a better understanding of the most important scattering objects and might help improving geometry-based stochastic MIMO models as in [9], [10] and [11]. 


\section{MEASUREMENTS}

\section{A. Measurement Equipment}

The radio channel data was collected using the HHI Channel Sounder [12]. This measurement device comes with $2 \times 3$ (two at Tx, three at Rx) fully parallel MIMO frontends and operates at $5.7 \mathrm{GHz}$ with a bandwidth of $1 \mathrm{GHz}$. The channel sounder consists of a Tx and a Rx part, which are both placed in conventional minivans. For these measurements, we used 4 out of the available 6 MIMO channels and mounted omnidirectional and vertically polarized antennas on the roof at the left and right edges of the vehicles. In order to record the position of the vehicles during measurements, we installed a positioning system, GeneSys ADMA-G. This highly accurate system works reliably even in deep street canyons, where coverage of GPS satellites is limited. The measurements were accompanied by conventional and $360^{\circ}$ video cameras mounted on the roof of both vehicles.

\section{B. Measurement Scenario}

The measurements were conducted in Berlin at the crossing of Pestalozzistr and Schlueterstr; details and GPS coordinates can be found in Table 1 in [3]. This crossing is a 4-leg intersection with street widths of $21 \mathrm{~m}$ on the east-west street and $33 \mathrm{~m}$ on the north-south street. All four corners have buildings relatively close to the intersection center, a communication scenario that accounts for about $50 \%$ of German urban intersections according to [13]. The two corners on the east-side of the intersection have a regular rectangular shape, while the two corners on the west-side are truncated with a $45^{\circ}$-wall of around $4 \mathrm{~m}$ length. On the sidewalk in front of each building corner, there are on average three traffic signs that could act as scattering objects. Also, parked or moving vehicles, especially vehicles with larger metallic surfaces might act as scatterers and increase the received power.

Naturally, two vehicles on collision course will at some point see each other and change from NLOS to the LOS. Since communication under LOS conditions yields a very high PDR (see [3] for results on this) and the operability of other complementary vehicular sensors, we only consider the NLOS phase and take the NLOS/LOS transition line as our reference point (see [3] for additional explanations). In Fig. 1 the transition line for each vehicle trajectory is marked as $0 \mathrm{~m}$, while $-30 \mathrm{~m}$ marks the distance to this reference point. The distance between the NLOS/LOS transition line and the crossing center is $32-38 \mathrm{~m}$ for the north-south road and 22-27 $\mathrm{m}$ for the east-west road. In order to validate the accuracy of the positioning system, we plotted the strongest path under LOS condition (the measurement phase we disregarded for our results), translated the path delay into meters and compared the results to the ADMA positioning system results. A maximum difference of $2 \mathrm{~m}$ could be observed between the two independent systems, which gave us confidence in the

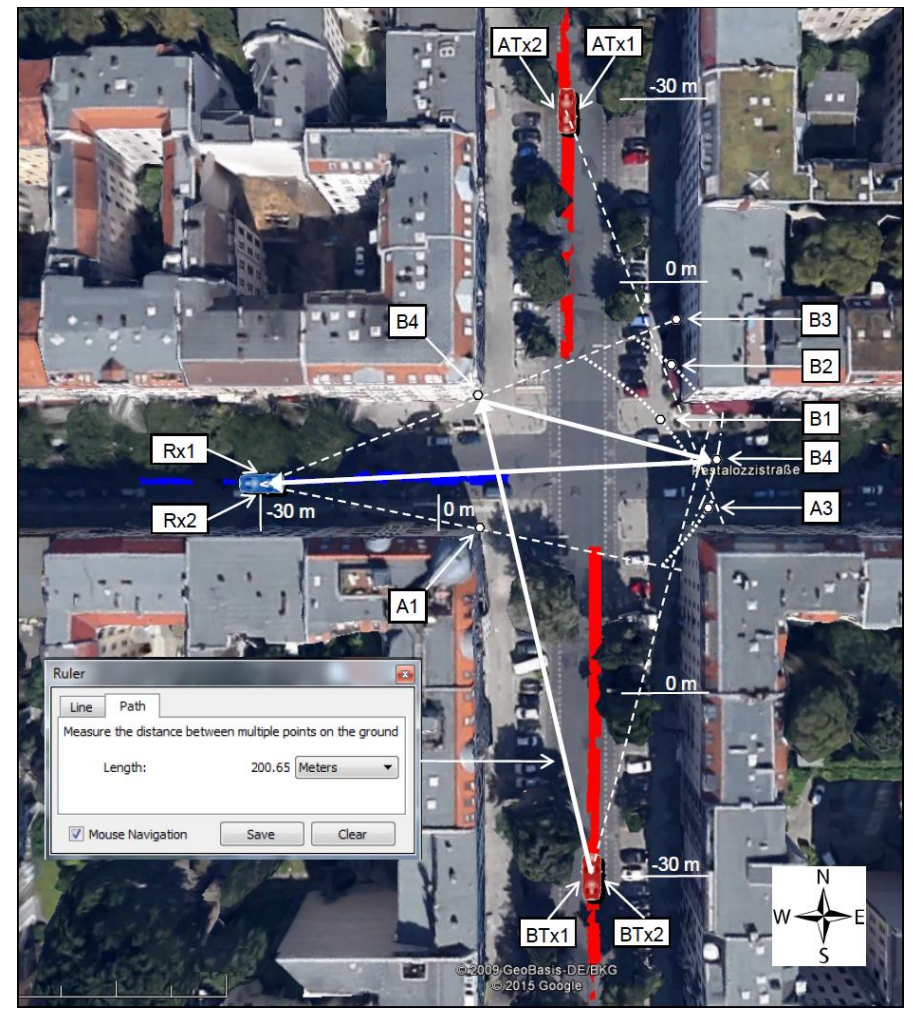

Figure 1. Overview of measurement runs and hypothesis of scatterer positions

accuracy of the positioning results. In order to avoid a complicated analysis w.r.t. the two-dimensional distance to the NLOS/LOS transition line as in [3], we selected measurement runs with similar speeds and similar distances to the center of the intersection for both vehicles, and used the mean distance of both vehicles for further analysis. Fig. 1 shows an overview of the two investigated measurement runs: run $A$ with the transmitter driving from the north towards the intersection and measurement run $B$ with the transmitter coming from the south. The average vehicle speed was $30-38 \mathrm{~km} / \mathrm{h}$, i.e. around $10 \mathrm{~m} / \mathrm{s}$.

\section{DATA PROCESSING}

\section{A. Preprocessing of Channel Sounder Data}

The recorded channel data consists of channel impulse responses at certain sampling instances, the so-called snapshots, which are organized in sequences of snapshots, the so-called sets. The duration of each measurement run is about 7.7 s. A measurement run consists of 1397 snapshots per set and 7 sets per measurement, which yields to a total of 9779 snapshots per measurement. The time gap between two snapshots is $716 \mu \mathrm{s}$. Between the sets there is a $100 \mathrm{~ms}$ time gap to allow the automatic gain control to measure and adapt to the current received power level. The recorded radio channel data is processed in multiple steps in order to extract the targeted MPC information. The data processing starts with identifying the noise parts of the channel impulse response and setting it to zero. This is done by a noise floor level estimation 


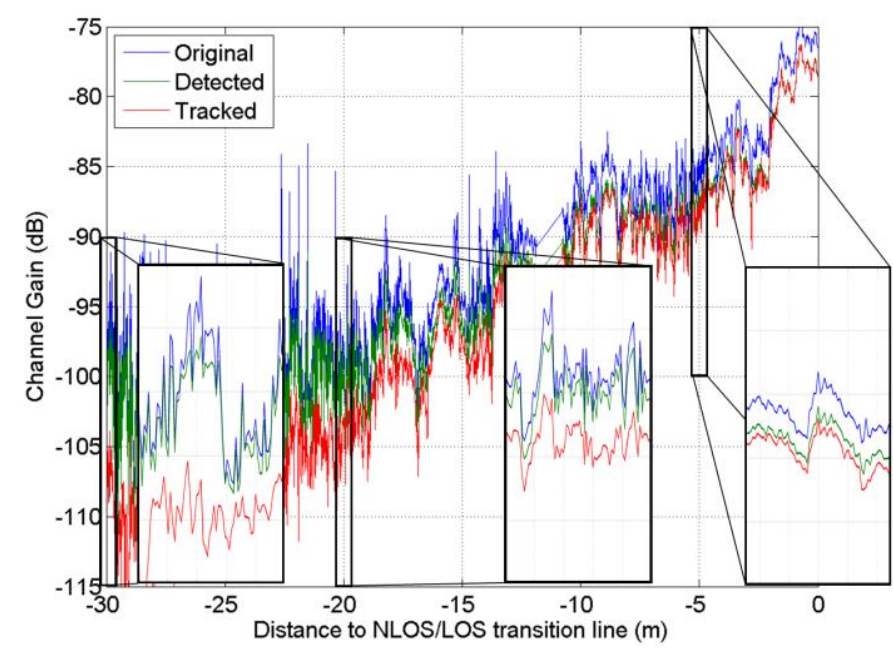

Figure 2. Comparison of channel gain at different stages of data processing

and a threshold of $6 \mathrm{~dB}$ above this noise floor is used (see Fig. 5 in [14] for details). The next processing step is a high delay resolution MPC detection method developed for UWB channel data and presented in [15]. The main idea of this method is based on the detection of the strongest MPC, a subtraction of this MPC from the channel data and a search for the next strongest MPC. This method was adapted to our measurement data and tested with an artificial channel data set including known MPCs. Finally, the method worked remarkably well with highly accurate sub-nanosecond MPC delay estimates. The next processing step involved a tracking method of the time evolution of the extracted MPCs, i.e. the connection of MPCs across adjacent snapshots. Due to the limited space in this paper, we leave the detailed description of this tracking method and its comprehensive performance evaluation for future publications. Basically, the tracking method takes advantage of the high measurement bandwidth, the predictability of the geometry and the known limits of the movement of the scattering objects. A thorough evaluation of the MPC tracking method and its performance has been carried out. One performance indicator is the ability of the method to track the channel gain. Fig. 2 shows the original channel gain, the detected channel gain (based on [15]) and the tracked channel gain (based on [15] and the MPC tracking method) for measurement run A.

It can be observed that both processed channel gain curves, the detected and the tracked curves, are below the original channel gain curve and follow the original curve progression reasonably well with a certain distance. As the vehicles get closer to the crossing center, the distance between the curves decreases and the characteristics become more alike. For the considered interval of distances to the NLOS/LOS transition line in Fig. 2, the mean offset between the original and detected channel gain is around $2 \mathrm{~dB}$, while the mean distance between detected and tracked channel gain is also around $2 \mathrm{~dB}$.

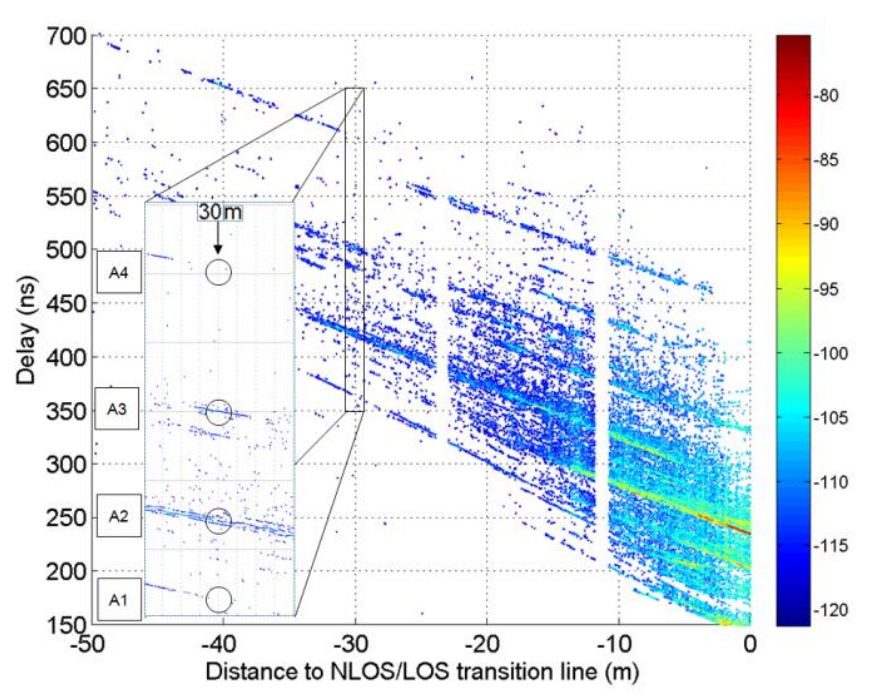

Figure 3. Delay of MPCs vs. distance of MIMO channel Tx1Rx1 in measurement run A (ATx1 to Rx1 in Fig. 1)

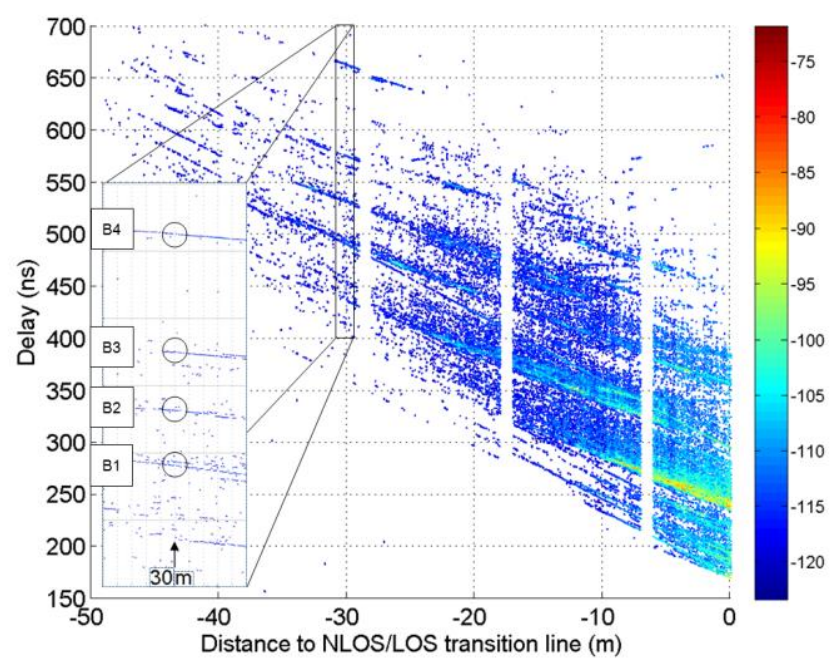

Figure 4. Delay of MPCs vs. distance of MIMO channel Tx2Rx1 in measurement run B (BTx2 to Rx1 in Fig. 1)

\section{RESULTS}

\section{A. Channel Parameters}

Important parameters of the vehicular radio channel are channel gain, Doppler spread and delay spread. In our measurements, the averaged channel gain has a slope of about $1 \mathrm{~dB} / \mathrm{m}$ : the measured channel gain at $40 \mathrm{~m}$ distance to NLOS/LOS transition line is around $-120 \mathrm{~dB}$, at the NLOS/LOS transition line it is found to be about $-80 \mathrm{~dB}$. The RMS Doppler spread is usually around $200 \mathrm{~Hz}$, with higher values of up to $600 \mathrm{~Hz}$. The RMS delay spread is usually around $40 \mathrm{~ns}$ with peaks of up to $60 \mathrm{~ns}$ at larger distances to the NLOS/LOS transition line. The measured values for the delay and Doppler spread are in accordance with values found for an urban intersection measurement in Fig. 4 in [16]. 


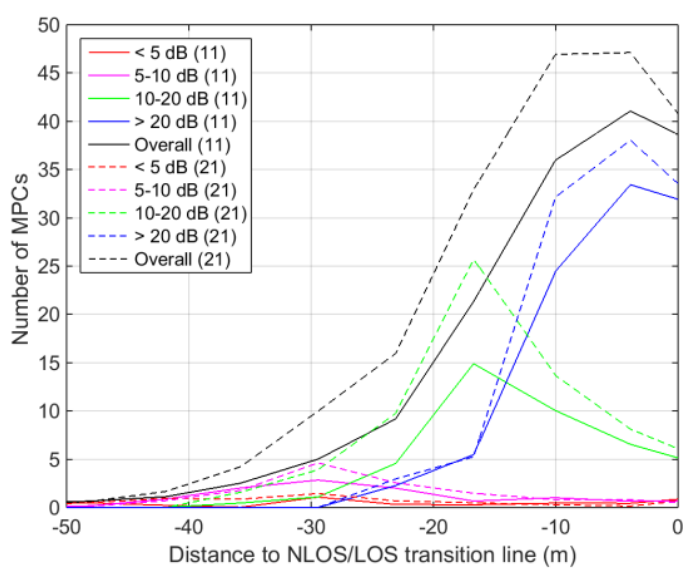

Figure 5. Measurement A: bins for number of MPCs for two MIMO channels

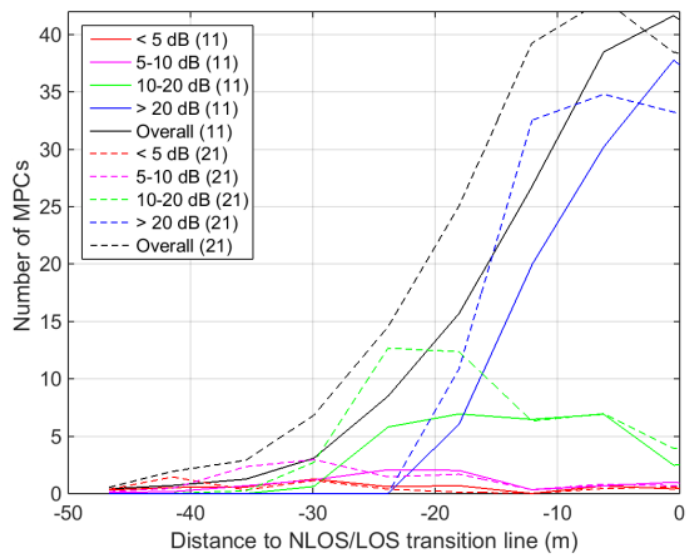

Figure 6. Measurement B: bins for number of MPCs for two MIMO channels

\section{B. Scatterer localization}

For the analysis of scattering points we here focus on the propagation process at $30 \mathrm{~m}$ distance, since this stage can be viewed as a region where communication starts and reaches a PDR level of around 50\% (see [3]). We took the delay time of the MPCs for both measurement runs (A1-A4 in Fig. 3 and B1-B4 in Fig. 4), computed the corresponding MPC travel distance and used a GoogleEarth ${ }^{\mathrm{TM}}$ tool called "Path Ruler" to test propagation hypothesis with good accuracy (lower left corner in Fig. 1). This method is basically equivalent to drawing a scattering ellipse. The visibility limitations for single-bounce reflections (dashed line in Fig. 1) led to the hypothesis of scatterer position A1, A3 and B1-B3 in Fig. 1.

The possible scatterer positions of B1-B3 are straightforward and it can be observed that a MPC with a delay time larger than B3 has to be a double-bounce reflection path. The most likely path for B4 is a reflection at the northwest truncated corner and a second reflection at an object on the eastern part of the intersection as indicated with the continuous arrow in Fig. 1. The travel distance of A1 fits very well with the position of the south-west corner of the intersec-

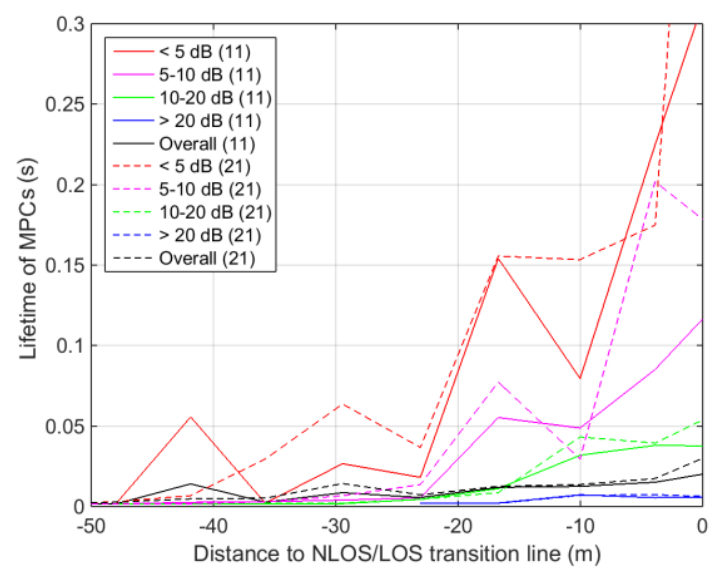

Figure 7. Measurement A: bins for lifetime of MPCs for two MIMO channels

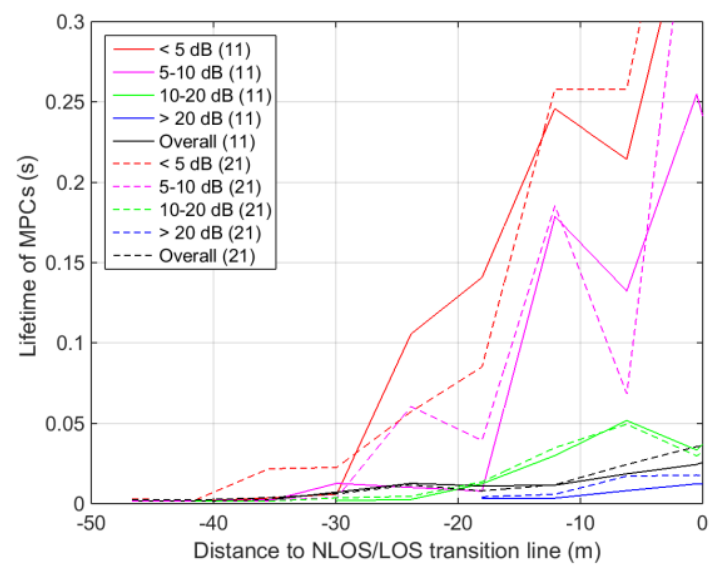

Figure 8. Measurement B: bins for lifetime of MPCs for two MIMO channels

tion. The most likely path for A2 is a double bounce reflection with incident angles equal to emergent angles at the eastern wall of the north-south road and the southern wall of the eastwest road. This hypothesis is also supported by the delay dispersion in Fig. 3. The travel distance of A4 leads to a similar propagation path hypothesis as for B4, but mirrored on the east-west axis: a first reflection at point $\mathrm{A} 1$ and a second reflection at a scattering object in the eastern part of the intersection.

\section{MPC Characteristics}

For the following analysis we grouped the MPCs of the entire measurement into four groups, based on their dynamic ranges (the power distances to the strongest $\mathrm{MPC}$ ): $<5 \mathrm{~dB}, 5$ $10 \mathrm{~dB}, 10-20 \mathrm{~dB}$ and above $20 \mathrm{~dB}$ dynamic range. Due to the high measurement bandwidth, we assume that we are able to resolve so-called physical paths, i.e. paths that are not superimposed by other paths and therefore do not undergo amplitude fluctuations due to constructive or destructive superposition. In order to show that this assumption is valid to some extent, we looked at the standard deviation of the MPC power and found that a typical value was around $2 \mathrm{~dB}$, with 
larger deviations of up to $4 \mathrm{~dB}$ for larger MPCs and smaller distances.

The analysis was done for all four MIMO channels, but due to limited space we only can show results for the channels 11 (Tx to Rx1) and 21 (Tx2 to Rx1). As can be seen in Fig. 5 and Fig. 6, the overall number of MPC starts rising at a distance of $40 \mathrm{~m}$ and reaches a number of 40-50 MPCs at distances below $10 \mathrm{~m}$. The number of MPC in the group > $20 \mathrm{~dB}$, and consequently the overall number of MPCs, drops shortly before the NLOS/LOS transition line. This is probably due to the increased energy at this distance, the limited dynamic range of the channel sounder. At smaller distances, the groups with larger dynamic ranges dominate, while at larger distances the influence of the dynamic range thresholds disappear in the noise floor and the overall number of MPCs is only composed of MPCs from smaller dynamic range groups. The drop for very short distances to the NLOS/LOS transition line was not observed for the MIMO channels 11 and 12 of measurement run $\mathrm{B}$, which could be explained with the position of $\mathrm{Tx} 1$ and the delayed line-of-sight to both $\mathrm{Rx}$ antennas.

The lifetime of MPCs strongly depends on the dynamic range group, since weak MPCs seem to have a shorter lifetime. As can be seen in Fig. 7 and Fig. 8, the lifetime of $<5$ $\mathrm{dB}$ dynamic range MPCs peaks at distances between 10-20 m, drops again at distances below $10 \mathrm{~m}$ and rises up to a lifetime of $0.3 \mathrm{~s}$ at the NLOS/LOS transition line, which corresponds to $3 \mathrm{~m}$ travel distance of the vehicles. This behavior can be observed for all MIMO channels in both measurement runs. The lifetime of the 5-10 dB dynamic range MPCs exhibits a similar progression with a generally shorter lifetime. For MIMO channel 11 in measurement run $A$, a peak can be observed for the $<5 \mathrm{~dB}$ dynamic range MPCs at around $40 \mathrm{~m}$, which can be explained in Fig. 3 with the presence of the scatterer A4.

\section{SUMMARY}

This paper introduces an analysis of wideband channel sounder data for inter-vehicular communication at an urban intersection. We set up hypothesis for scatterer positions and found that single-bounce scatterers are located at or around building corners. Double-bounce reflections were found for roadside buildings parallel to the two vehicles and for reflections over truncated building corners.

A MPC tracking algorithm with good energy tracking performance was applied. Based on this method, the progression of the number of MPCs and the lifetime of MPCs for different MPC dynamic range groups could be shown. To the knowledge of the authors, this is the first analysis on the number of and lifetime of MPCs at an urban intersection and might be beneficial improving geometry-based stochastic MIMO channel models.

\section{REFERENCES}

[1] Schumacher, H.; Tchouankem, H.; Nuckelt, J.; Kurner, T.; Zinchenko, T.; Leschke, A.; Wolf, L., "Vehicle-to-Vehicle IEEE 802.11p performance measurements at urban intersections," Communications (ICC), 2012 IEEE International Conference on , 10-15 June 2012

[2] Mangel, T.; Michl, M.; Klemp, O; Hartenstein, H. "Real-world measurements of non-line-of-sight reception quality for 5.9GHz IEEE 802.11p at intersections." In Proceedings of the Third international conference on Communication technologies for vehicles (Nets4Cars/Nets4Trains'11), Thomas Strang, Cristina Rico Garcia, Andreas Festag, Alexey Vinel, and Rashid Mehmood (Eds.). Springer-Verlag, Berlin, Heidelberg, 189202. 2011.

[3] Mahler, K.; Paschalidis, P.; Wisotzki, M.; Kortke, A.; Keusgen, W., "Evaluation of Vehicular Communication Performance at Street Intersections," Vehicular Technology Conference (VTC Fall), 2014 IEEE 80th , 14-17 Sept. 2014

[4] Karedal, J.; Tufvesson, F.; Abbas, T.; Klemp, O.; Paier, A.; Bernado, L.; Molisch, A.F., "Radio Channel Measurements at Street Intersections for Vehicle-to-Vehicle Safety Applications," Vehicular Technology Conference (VTC 2010-Spring), 2010 IEEE 71st , 16-19 May 2010

[5] Abbas, T.; Karedal, J.; Tufvesson, F.; Paier, A.; Bernado, L.; Molisch, A.F., "Directional Analysis of Vehicle-to-Vehicle Propagation Channels," Vehicular Technology Conference (VTC Spring), 2011 IEEE 73rd , 15-18 May 2011

[6] Nuckelt, J.; Abbas, T.; Tufvesson, F.; Mecklenbrauker, C.; Bernado, L.; Kurner, T., "Comparison of Ray Tracing and Channel-Sounder Measurements for Vehicular Communications," Vehicular Technology Conference (VTC Spring), 2013 IEEE 77th , 2-5 June 2013

[7] Abbas, T.; Thiel, A.; Zemen, T.; Mecklenbrauker, C.F.; Tufvesson, F., "Validation of a non-line-of-sight path-loss model for V2V communications at street intersections," ITS Telecommunications (ITST), 2013 13th International Conference on , 5-7 Nov. 2013

[8] Mangel T., Klemp O., and Hartenstein H., "5.9 GHz inter-vehicle communication at intersections: a validated non-line-of-sight path-loss and fading model," EURASIP J. Wireless Commun. Networking, vol. 2011, no. 1, 2011.

[9] Karedal, J.; Tufvesson, F.; Czink, N.; Paier, A.; Dumard, C.; Zemen, T.; Mecklenbrauker, C.F.; Molisch, A.F., "A geometry-based stochastic MIMO model for vehicle-to-vehicle communications," Wireless Communications, IEEE Transactions on , vol.8, no.7, July 2009

[10] Theodorakopoulos, A.; Papaioannou, P.; Abbas, T.; Tufvesson, F., "A geometry based stochastic model for MIMO V2V channel simulation in cross-junction scenario," ITS Telecommunications (ITST), 2013 13th International Conference on , 5-7 Nov. 2013

[11] Zhinan Xu; Bernado, L.; Mingming Gan; Hofer, M.; Abbas, T.; Shivaldova, V.; Mahler, K.; Smely, D.; Zemen, T., "Relaying for IEEE $802.11 \mathrm{p}$ at road intersection using a vehicular non-stationary channel model," Wireless Vehicular Communications (WiVeC), 2014 IEEE 6th International Symposium on , 14-15 Sept. 2014

[12] Paschalidis, P.; Wisotzki, M.; Kortke, A.; Keusgen, W.; Peter, M.; , "A Wideband Channel Sounder for Car-to-Car Radio Channel Measurements at $5.7 \mathrm{GHz}$ and Results for an Urban Scenario," Vehicular Technology Conference, 2008. VTC 2008-Fall. IEEE 68th , 21-24 Sept. 2008

[13] Mangel, T.; Schweizer, F.; Kosch, T.; Hartenstein, H., "Vehicular safety communication at intersections: Buildings, Non-Line-Of-Sight and representative scenarios," Wireless On-Demand Network Systems and Services (WONS), 2011 Eighth International Conference on , 26-28 Jan. 2011

[14] Mahler, K.; Paschalidis, P.; Kortke, A.; Peter, M.; Keusgen, W., "Realistic IEEE 802.11p Transmission Simulations Based on Channel Sounder Measurement Data," Vehicular Technology Conference (VTC Fall), 2013 IEEE 78th , 2-5 Sept. 2013

[15] Santos, T.; Karedal, J.; Almers, P.; Tufvesson, F.; Molisch, A.F., "Scatterer Detection by Successive Cancellation for UWB - Method and Experimental Verification," Vehicular Technology Conference, 2008. VTC Spring 2008. IEEE , 11-14 May 2008

[16] Bernado, L.; Zemen, T.; Tufvesson, F.; Molisch, A.F.; Mecklenbrauker, C.F., "Delay and Doppler Spreads of Nonstationary Vehicular Channels for Safety-Relevant Scenarios," Vehicular Technology, IEEE Transactions on , vol.63, no.1, Jan. 2014 